\section{ORIGINAL RESEARCH}

\author{
S.H. Suh \\ B.M. Kim \\ H.G. Roh \\ K.-Y. Lee \\ S.I. Park \\ D.I. Kim \\ D.J. Kim \\ H.S. Nam \\ H.S. Choi
}

\title{
Self-Expanding Stent for Recanalization of Acute Embolic or Dissecting Intracranial Artery Occlusion
}

BACKGROUND AND PURPOSE: Stent placement may be an effective and last resort method for recanalization of recalcitrant intracranial artery occlusion. The purpose of this study was to evaluate the safety and efficacy of a self-expanding stent for the recanalization of acute embolic or dissecting intracranial artery occlusion.

MATERIALS AND METHODS: Nine patients (mean age, 66 years; NIHSS score, 10-23) with acute embolic $(n=8)$ or dissecting occlusion $(n=1)$ of the intracranial arteries (ICA terminus in 5, MCA in 3 , and BA in 1) were treated with a recapturable self-expanding stent. The safety and efficacy of the stent for recanalization were evaluated retrospectively.

RESULTS: The emboli were entrapped against the vessel wall by the stent, resulting in immediate recanalization (TIMI 2) in all embolic occlusions. The dissecting occlusion was recanalized completely (TIMI 3). Adjunctive thrombolytics ( $n=8$, urokinase, 100,000-300,000 U) and/or GP Illb/llla antagonist ( $n=7$, tirofiban, $0.5-1 \mathrm{mg}$ ) were administered intra-arterially, and the degree of recanalization further improved in 4 embolic occlusions (TIMI 3). Acute in-stent thrombosis occurred in 2 patients, who received only urokinase without GP IIb/Illa antagonist. Both of the reoccluded arteries were reopened, by stent recapture in 1 and by intra-arterial administration of GP IIb/lla antagonist in the other. Recapture was attempted in 7 cases, of which there were 3 successful outcomes. There was 1 asymptomatic hemorrhagic conversion at the infarction site. The mean improvement of the NIHSS score between baseline and discharge was 12.3 (range, 3-22).

CONCLUSIONS: Preliminary results of this study suggest that a self-expanding stent may be safe and efficient for recanalization of acute embolic or dissecting intracranial artery occlusion.

\begin{abstract}
ABBREVIATIONS: $\mathrm{ACA}=$ anterior cerebral artery; $\mathrm{BA}=$ basilar artery; $\mathrm{Fx}=$ fracture; $\mathrm{GP}=$ glycoprotein; hrs = hours; ICA = internal carotid artery; IV = intravenous; M1 = MCA M1 portion; M2 = MCA M2 portion; MCA = middle cerebral artery; NIHSS = National Institutes of Health Stroke Scale; $\mathrm{PCA}=$ posterior cerebral artery; rtPA = recombinant tissue plasminogen activator; $\mathrm{TIMI}=$ Thrombolysis In Myocardial Ischemia; Tx = treatment; UK = urokinase
\end{abstract}

S tent placement may be an effective and last resort method for recanalization of recalcitrant intracranial artery occlusion with a published recanalization rate between $79 \%$ and $89 \% .{ }^{1-3}$ However, balloon-expandable stents are difficult to navigate through an intracranial artery and carry inherent risks associated with balloon inflation for stent deployment. Also, tortuosity and the difference in diameters of the parent artery proximal and distal to the occlusion site may make it difficult to apply balloon-expandable stents to the recanaliza-

\section{Received June 21, 2009; accepted after revision August 6.}

From the Departments of Radiology (S.H.S.) and Neurology (K.-Y.L.), Gangnam Severance Hospital, Yonsei University College of Medicine, Seoul, Korea; Departments of Radiology (B.M.K., D.I.K., D.J.K., H.S.C.) and Neurology (H.S.N.), Severance Hospital, Yonsei University College of Medicine, Seoul, Korea; Department of Radiology (H.G.R.), Konkuk Univeristy Hospital, Seoul Korea; and Department of Radiology (S.I.P.), Bucheon Hospital, Sooncheonhyang University, Bucheon, Korea.

This work was supported by a grant of the Korea Healthcare Technology R\&D Project, Ministry for Health, Welfare and Family Affairs, Republic of Korea (No. A085136).

Please address correspondence to Byung Moon Kim, MD, Department of Radiology, Severance Hospital, Yonsei University College of Medicine, Seoul, Republic of Korea; e-mail: bmoon21@hanmail.net

Indicates open access to non-subscribers at www.ajnr.org

Indicates article with supplemental on-line table.

DOI 10.3174/ajnr.A1865 tion of an acutely occluded intracranial artery. Recently, there were 2 reports regarding the use and efficacy of the self-expanding stent for the recanalization of acute intracranial artery occlusion., ${ }^{2,3}$ Also, 2 recent case reports described an innovative temporary endovascular bypass method for recanalization by using a newly developed Enterprise recapturable self-expanding stent (Cordis, Miami Lakes, Florida). ${ }^{4,5}$ However, little is known about the feasibility, safety, efficacy, and recapturability of the newly developed Enterprise stent for recanalization in acute strokes. In this case series, we present our experience in applying Enterprise stents to the recanalization of major intracranial arteries with acute embolic or dissecting occlusion.

\section{Materials and Methods}

The institutional review board approved this retrospective study. Patient informed consent for study inclusion was waived, though patient informed consent for pharmacologic or mechanical thrombolytic treatment, including angioplasty and/or stent placement, was obtained from the patients' legal representatives. Nine patients (mean age, 66 years; NIHSS score, $10-23)$ with acute embolic $(n=8)$ or dissecting $(n=1)$ occlusion of intracranial arteries were treated with Enterprise stents between November 2008 and April 2009. The patients had experienced an acute stroke within 12 hours of the symptom onset (range, 1.5-12 hours; mean, 5.3 hours) and had a baseline 
NIHSS score of $\geq 10$ (range, 10-23; mean, 17). There was no intracranial hemorrhage or clear early cerebral infarction that was more than or equal to one-third of the vessel distribution on the pretreatment CT and/or MR imaging. Acute occlusion of a major intracranial artery was identified on the pretreatment CT or MR angiography. The cause of the occlusion was judged according to pre-existing medical conditions and angiographic findings (On-line Table). IV administration of rtPA was not indicated because of the limited time window ( $>3$ hours after symptom onset), age older than 80 years, recent cardiac surgery, and/or traumatic arterial dissection, except in 1 patient who received a bridging dose of $\mathrm{rtPA}(0.5 \mathrm{mg} / \mathrm{kg})$ (On-line Table). The degree of recanalization was assessed by a neuroradiologist who did not participate in the treatment, in accordance with the TIMI grading system. Early clinical outcomes were evaluated according to the NIHSS score 24 hours after treatment and again at discharge.

Routine 3- or 4-vessel cerebral angiography was first performed before treatment. After the occlusion site of a major intracranial artery was identified, a 6F guiding catheter (Envoy, Cordis; or Shuttle, Cook, Bloomington, Indiana) was placed at the distal cervical portion of the relevant ICA or vertebral artery. After the placement of the guiding catheter, a 3000-unit IV heparin bolus was injected, followed by a $1000-$ unit/h IV infusion. Enterprise stent placement was the initial mechanical maneuver for the recanalization of acutely occluded vessels. The lesion length was estimated as the distance between the vessel cutoff on the baseline conventional angiography and the beginning of the normal vessel distal to the clot on the microcatheter angiography. A Prowler Select Plus microcatheter (Cordis) was navigated to a branch of the distal normal portion of the artery by using a 0.014-inch guidewire. Then an Enterprise stent was introduced and deployed, spanning the entire obstructive segment.

Enterprise stents were first partially deployed in 7 patients for possible recapture after recanalization. In the other 2 patients, Enterprise stents were fully deployed due to traumatic arterial dissection in 1 and for a second stent placement with a Y-configuration at the MCA bifurcation in the other. Immediately after stent deployment, 100,000 $\mathrm{U}$ of urokinase and a bolus of GP IIb/IIIa antagonist (tirofiban, 0.5-1 $\mathrm{mg}$ ) were loaded intra-arterially in 6 patients. Of the remaining 3 patients, 1 received GP IIb/IIIa antagonist (tirofiban, $0.5 \mathrm{mg}$ ) and the other 2 received 200,000 and 300,000 U of urokinase, respectively. GP IIb/IIIa antagonist was administrated due to the lack of antiplatelet premedication, and urokinase was infused for dissolving the embolus entrapped by the stent. In the patients with permanent stent placement, IV infusion of GP IIb/IIIa antagonist (tirofiban) was maintained for 24-36 hours after the completion of the procedure. In addition, a loading dose of dual antiplatelet medication (aspirin, 100$500 \mathrm{mg}$, plus clopidogrel, $300 \mathrm{mg}$ ) was immediately administered through an orally placed gastric tube or just orally, after the procedure. The maintenance of the dual antiplatelet medication dose was followed thereafter.

\section{Results}

The baseline clinical data, intervention, and early clinical and angiographic outcomes are all summarized in the On-line Table. Four patients had acute occlusion from the ICA terminus to both the proximal MCA and the ACA, 1 had an acute occlusion from the ICA terminus to the proximal MCA M1, 2 had acute occlusions from MCA M1 to the M2 portion, 1 had an acute occlusion at the MCA M1 portion, and 1 had acute occlusion from the distal BA to the bilateral PCAs. Seven patients had atrial fibrillation and revealed an abrupt cutoff from the normal-calibered artery on the angiogram. Therefore, the cause of acute arterial occlusion was judged to be a cardiogenic embolism. One patient had been admitted due to head trauma 2 days before the symptom onset and had a fracture of the ipsilateral optic strut adjacent to the occlusion site of the ICA; therefore, the cause of the occlusion was judged to be arterial dissection. The remaining patient had concomitant acute tandem occlusion of the ipsilateral cervical ICA bulb and MCA; therefore, the cause of the MCA occlusion was judged to be an artery-to-artery embolism.

The emboli were entrapped against the vessel wall by the Enterprise stent, resulting in immediate recanalization (TIMI 2) in all 8 embolic occlusions (Fig 1). The ICA dissecting occlusion was completely recanalized (TIMI, 3). After an adjunctive thrombolytic or a bolus of intra-arterial GP IIb/IIIa inhibitor, or both were administered, the degrees of recanalization were further improved to TIMI 3 in 4 embolic occlusions. In 2 of the remaining 4 embolic occlusions, the degree of recanalization was also improved but did not reach TIMI 3. The last 2 patients, who only received urokinase without the GP IIb/IIIa antagonist, revealed acute in-stent thrombosis 10-20 minutes after initial recanalization. Both of the re-occluded arteries in those 2 patients were reopened by recapture of the stent in 1 and by intra-arterial administration of GP IIb/IIIa antagonist (Fig 2) in the other.

Recapture was attempted in 7 patients in whom Enterprise stents were partially deployed. Recaptures were not attempted until the entrapped emboli were sufficiently resolved on the angiogram in 6 , with the exception of 1 patient in whom recapture was attempted due to acute in-stent occlusion. Therefore, the period between the initial partial deployment and the attempt to recapture varied from 15 to 180 minutes, according to the embolus burden. Recapture was successful in 3 patients in whom it was attempted within 30 minutes after partial deployment of the stent, whereas in the other 4 patients with recapture failure, it was attempted in $\geq 120$ minutes. There was only 1 small asymptomatic hemorrhagic conversion of the infarction site of the basal ganglia in a patient with traumatic ICA dissection. Mean improvement of the NIHSS score between the baseline and discharge was 12.3 (range, 3-22). Short-term follow-up vascular imaging (conventional angiography in 5 and CT and/or MR angiography in 4), which was available 3 days to 2 weeks after treatment, revealed persistent patency of the arteries in all patients and full expansion of the stent in the 6 patients who had undergone permanent stent placement (Fig 1).

\section{Discussion}

A recapturable self-expanding stent can be used as either a permanent stent placement or an endovascular temporary bypass method to entrap thrombus against the vessel wall for recanalization in the acute occlusion setting of a major intracranial artery. ${ }^{4-7}$ This strategy of using a recapturable self-expanding stent may provide immediate recanalization of an acutely occluded artery. This early restoration of flow may more quickly promote endogenous or pharmacologic thrombolysis. If blood flow through the occlusion site is restored, as seen in patients in this series, the thrombus entrapped by the stent may dissolve due to the GP IIb/IIIa antagonist and/or a small amount of thrombolytics. Furthermore, once reperfu- 

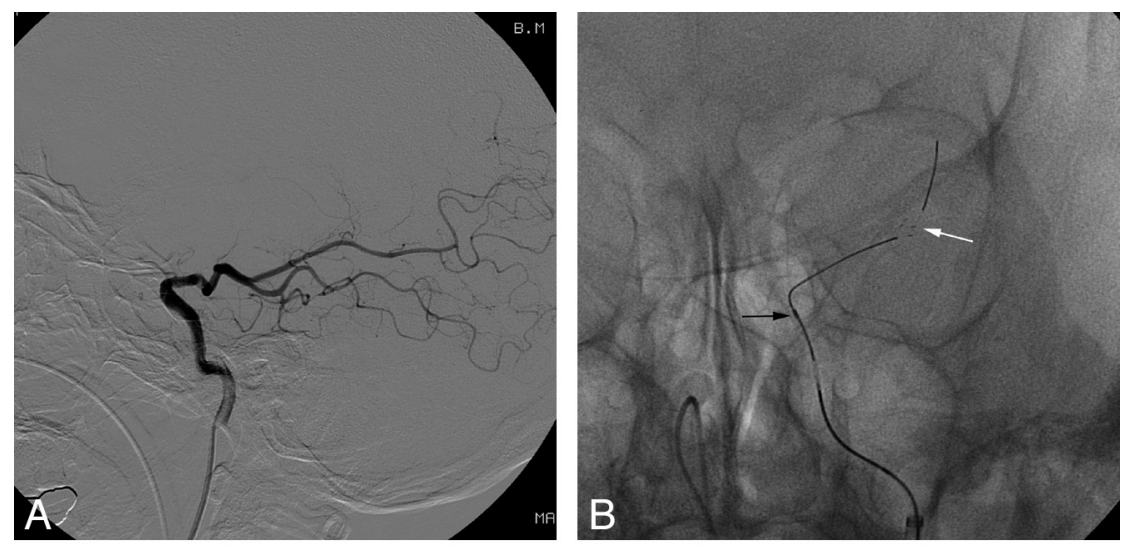

Fig 1. Case 1. A 44-year-old woman who presented with acute stroke and an NIHSS score of 22. A, Left internal carotid angiogram in the lateral projection shows acute cutoff just above the takeoff of the posterior communicating artery. $B$, Partial deployment of the Enterprise stent. Note the distal markers of the stent (white arrow) and the distal marker of the Prowler Select Plus microcatheter (black arrow). $C$, Three-hour delay angiogram in the lateral projection after the intra-arterial administration of urokinase $(100,000$ $\mathrm{U})$, and tirofiban $(0.5 \mathrm{mg})$, reveals a TIMI 3 recanalization. Note a notch (arrow) in the stent corresponding to the portion in which thrombus is entrapped between the stent and the arterial wall. D, Follow-up angiogram 1 week after thrombolysis reveals the full expansion of the previously noted notch in the stent and normal flow from the ICA to the MCA and ACA.

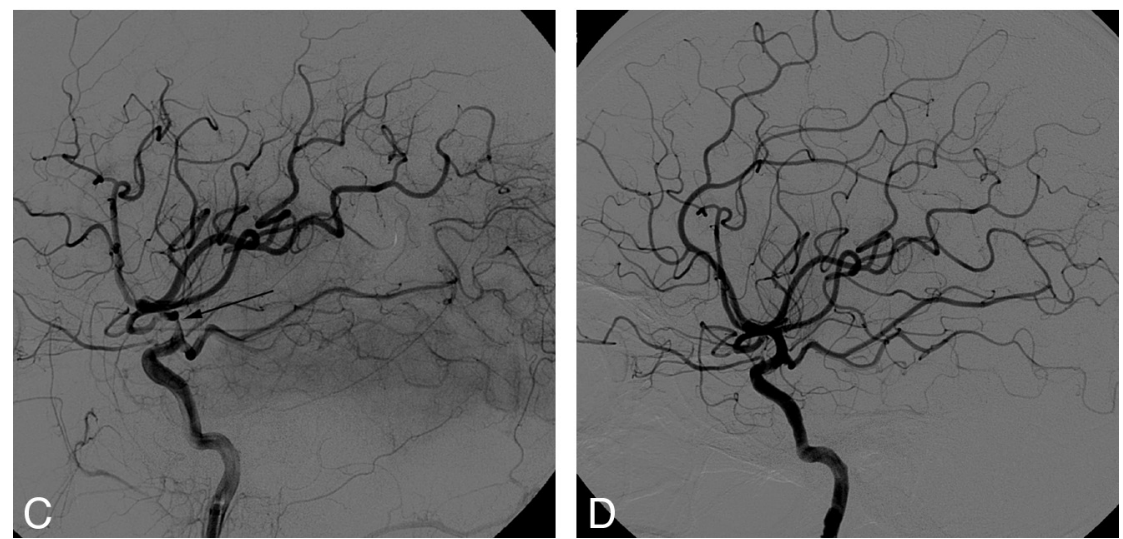

sion to the ischemic brain tissue is restored, it allows us enough time to preclude or to minimize the dose of thrombolytics to completely dissolve the remaining entrapped thrombus. A mean of 150,000 U (range, 100,000-300,000 U) of urokinase was used in 8 of 9 patients, which was a very small amount, considering the large amount of thrombus burden at the ICA terminus or proximal MCA. Moreover, in the remaining patient, only GP IIb/IIIa antagonist without thrombolytics was sufficient to prevent reocclusion and to dissolve the entrapped thrombus.

In the cardioembolic occlusion at the ICA terminus, recanalization is very difficult with intra-arterial strategies as well as IV rtPA because of the large amount of thrombus burden. The causes of the 5 ICA terminus occlusions included in our series consisted of 4 cardiogenic embolisms and 1 traumatic dissection. In all 4 cases of cardioembolic occlusion from the ICA terminus extending to both the proximal MCA and ACA, immediate recanalization could be achieved by partial deployment of the stent, despite a large amount of embolus burden. This immediate recanalization may be the most important advantage of the stent placement in acute stroke. Another possible advantage of the stent strategy for recanalization is to prohibit distal migration of the embolus. In our experience, distal migration of the embolus was frequently observed as the embolus dissolved and became smaller during intra-arterial thrombolysis with rtPA or urokinase; one example was the migration of the embolus from the ICA to the MCA. When applying mechanical thrombolysis by using a microwire or balloon, we also frequently observed distal migration/embolization of the embolus or macerated clots. Although the mac- erated emboli were relatively easily dissolved by thrombolytics or endogenously, it was sometimes problematic in that those emboli frequently occluded the arterial branches supplying the eloquent area of the brain. On the other hand, complete or partial deployment of a self-expanding stent spanning the entire embolus is less likely to cause distal migration or embolization of the embolus. In our patients, there was no distal migration or embolization of the entrapped embolus visible on the angiogram.

One may be concerned that a self-expanding stent may not have sufficient radial force to recanalize the occluded artery. However, initial recanalization was successful in all of our patients, including cardioembolic $(n=7)$, artery-to-artery embolic $(n=1)$, and arterial dissection $(n=1)$. This suggests that a self-expanding stent with low radial force may be sufficient to entrap the embolus against the vessel wall, causing immediate recanalization of acute embolic or dissecting occlusion.

There were only 2 case series regarding the use of self-expanding stents for recanalization in acute stroke in the existing literature, in which recanalization rates with self-expanding stents were $79 \%$ and $89 \%$, respectively. ${ }^{2,3}$ In the experimental embolism model, the TIMI $2 / 3$ recanalization rate with selfexpanding stents was $91 \% .^{8}$ Although it is impossible to directly compare our results and those of the previous 2 case series because of the small number of patients, we guess that the case selection may affect the rate of recanalization. In our patients, the cause of acute arterial occlusion was not arteriosclerotic stenosis but rather obviously emboli or dissection. In the previous 2 series, the causes of acute occlusion were not mentioned; therefore, those cases might have included arte- 

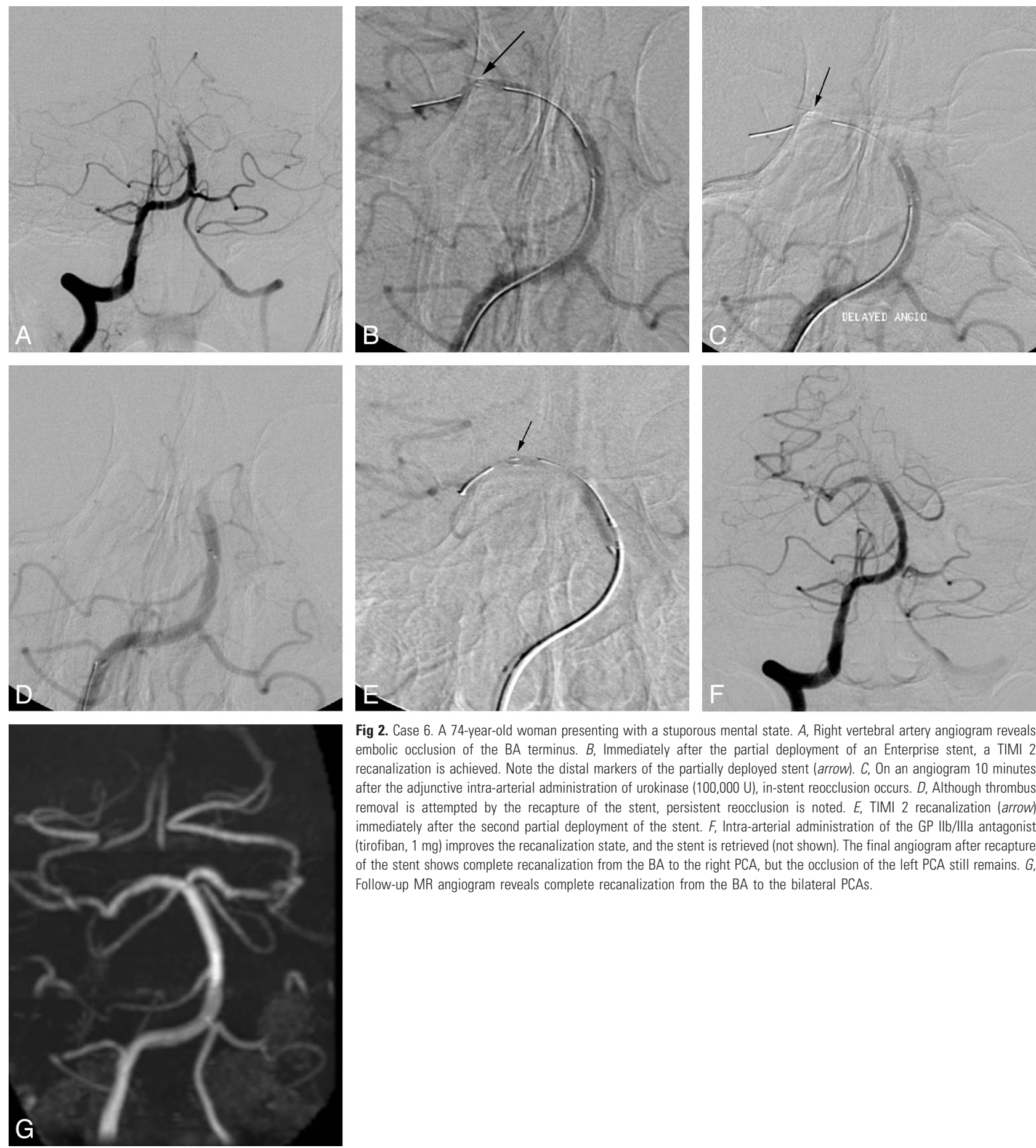

Fig 2. Case 6. A 74-year-old woman presenting with a stuporous mental state. $A$, Right vertebral artery angiogram reveals embolic occlusion of the BA terminus. B, Immediately after the partial deployment of an Enterprise stent, a TIMI 2 recanalization is achieved. Note the distal markers of the partially deployed stent (arrow). C, On an angiogram 10 minutes after the adjunctive intra-arterial administration of urokinase $(100,000 \mathrm{U})$, in-stent reocclusion occurs. $D$, Although thrombus removal is attempted by the recapture of the stent, persistent reocclusion is noted. E, TIMI 2 recanalization (arrow) immediately after the second partial deployment of the stent. $F$, Intra-arterial administration of the GP Ilb/llla antagonist (tirofiban, $1 \mathrm{mg}$ ) improves the recanalization state, and the stent is retrieved (not shown). The final angiogram after recapture of the stent shows complete recanalization from the BA to the right PCA, but the occlusion of the left PCA still remains. $G$ Follow-up MR angiogram reveals complete recanalization from the BA to the bilateral PCAs.

riosclerotic in situ occlusion at the pre-existing stenotic portion of the artery. In our opinion, a self-expanding stent may be less likely to be effective in the recanalization of acute arteriosclerotic in situ occlusion with pre-existing stenosis because of its low radial force.

Recapturability of the Enterprise stent is an important advantage in its use for recanalization of acute strokes. As mentioned in 2 innovative case reports, the recapture of the stent after thrombolysis has several possible advantages over permanent stent placement. ${ }^{4,5}$ In our case series, however, recapture was successful in 3 of the 7 attempted cases. One possible explanation is the difference in time from deployment to at- tempt to recapture between the successful and the failed cases. In the 4 failed cases, the time was $\geq 120$ minutes whereas the duration of our 3 successful procedures and the other 2 reported procedures ${ }^{4,5}$ was $\leq 30$ minutes. The longer the stent is partially deployed, the greater is the possibility of microclot deposition within the stent-delivery catheter. This may increase friction and difficulty during the recapture of the stent. In the acute phase of a stroke, 1 of the major concerns regarding the use of the stent is in-stent thrombosis due to the lack of antiplatelet premedication. In our series, in-stent thrombosis occurred in 2 patients, both of whom received only urokinase, not the GP IIb/IIIa antagonist. This suggests that the GP IIb/ 
IIIa antagonist may be essential in stent placement for the recanalization of an acute stroke with lack of antiplatelet premedication. Another major concern is the possibility of increased risk of fatal intracranial hemorrhage associated with procedural and/or postprocedural antiplatelet medication. Although only a small number of patients were included, there was no symptomatic intracranial hemorrhage in our series. The possible risk and efficacy of self-expanding stents for the recanalization of recalcitrant intracranial artery occlusion warrants further study.

\section{Conclusions}

Although a small number of patients were included in this study, preliminary results of this case series suggest that a selfexpanding stent may be a safe and efficient tool for recanalization of acute embolic or dissecting intracranial artery occlusion. These preliminary results may warrant further study regarding the application of this type of stent to acute embolic or dissecting intracranial artery occlusion within a larger population.

\section{References}

1. Levy EI, Ecker RD, Horowitz MB, et al. Stent-assisted intracranial recanalization for acute stroke: early results. Neurosurgery 2006;58:458-63

2. Levy EI, Mehta R, Gupta R, et al. Self-expanding stents for recanalization of acute cerebrovascular occlusions. AJNR Am J Neuroradiol 2007;28: $816-22$

3. Zaidat OO, Wolfe T, Hussain SI, et al. Interventional acute ischemic stroke therapy with intracranial self-expanding stent. Stroke 2008;39:2392-95

4. Kelly ME, Furlan AJ, Fiorella D. Recanalization of an acute middle cerebral artery occlusion using a self-expanding, reconstrainable intracranial microstent as a temporary endovascular bypass. Stroke 2008;39:1770-73. Epub 2008 Apr 3

5. Hauck EF, Mocco J, Snyder KV, et al. Temporary endovascular bypass: a novel treatment for acute stroke. AJNR Am J Neuroardiol 2009;30:1532-33. Epub 2009 Mar 11

6. Nogueira RG, Schwamm LH, Hirsch JA. Endovascular approach to acute stroke. Part 1. Drugs, devices, and data. AJNR Am J Neuroradiol 2009;30: 649-61

7. Chiam PT, Samuelson RM, Mocco J, et al. Navigability trumps all: stenting of acute middle cerebral artery occlusions with a new self-expandable stent. AJNR Am J Neuroradiol 2008;29:1956-58

8. Levy EI, Sauvaqeau E, Hanel RA, et al. Self-expanding versus balloon-mounted stents for vessel recanalization following embolic occlusion in the canine model: technical feasibility study. AJNR Am J Neuroradiol 2006;27:2069-72

9. Suh SH, Lee KY, Hong CK, et al. Temporary stenting and retrieval of the self-expandable, intracranial stent in acute middle cerebral artery occlusion. Neuroradiology 2009;51:541-44. Epub 2009 May 8 